\title{
Intensity variations in EIT shutterless mode: Waves or flows?
}

\author{
A. De Groof ${ }^{1}$, D. Berghmans ${ }^{2}$, L. van Driel-Gesztelyi ${ }^{1,3,4,5}$, and S. Poedts ${ }^{1}$ \\ 1 Centre for Plasma Astrophysics, K.U. Leuven, Celestijnenlaan 200 B, 3001 Leuven, Belgium \\ 2 Royal Observatory of Belgium, Ringlaan 3 Av. Circulaire, 1180 Brussels, Belgium \\ e-mail: david.berghmans@oma.be \\ 3 Mullard Space Science Laboratory, Holmbury St. Mary, Dorking, Surrey RH5 6NT, UK \\ 4 Observatoire de Paris, LESIA, FRE2461 (CNRS), 92195 Meudon Cedex, France \\ e-mail: lidia.vandriel@obspm. fr \\ 5 Konkoly Observatory, PO Box 67, 1525 Budapest, Hungary
}

Received 1 September 2003 / Accepted 3 November 2003

\begin{abstract}
On 11 July 2001 an EIT shutterless campaign was conducted which provided 120 high-cadence (68 s) $304 \AA$ A images of the north eastern quarter of the Sun. The most interesting feature seen in the data is an off-limb half loop structure along which systematic intensity variations are seen which appear to propagate from the top of the loop towards its footpoint. We investigate the underlying cause of these propagating disturbances, i.e. whether they are caused by waves or by plasma flows. First we identify 7 blobs with the highest intensities and follow them along the loop. By means of a location-time plot, bulk velocities can be measured at several locations along the loop. The velocity curve found this way is then compared with characteristic wave speeds and with the free-fall speed in order to deduce the nature of the intensity variations. Additional information on density and temperature is derived by measuring the relative intensity enhancements and comparing the EIT $304 \AA$ sequence with Big Bear data and $171 \AA$ data (TRACE/EIT). The combination of all these constraints gives us an insight on the nature and origin of these intensity variations. The idea of slow magneto-acoustic waves is rejected, and we find several arguments supporting that these intensity variations are due to flowing/falling plasma blobs.
\end{abstract}

Key words. Sun: atmosphere - Sun: magnetic fields - Sun: oscillations

\section{Introduction}

Propagating intensity variations in magnetic loop structures are often seen in the solar atmosphere and are of great importance for coronal seismology and for the understanding of coronal heating mechanisms. Some show a periodic behaviour and are most often classified as slow magneto-acoustic waves propagating along coronal loops. Extended reviews of the observations of various periodic and quasi-periodic oscillations are written by e.g. Aschwanden (1987) and Tsubaki (1988). Later, the high resolution images of SoHO and TRACE suddenly increased the number and detail of the investigations and many authors presented the characteristics of oscillations observed by the high-resolution imager TRACE (e.g. Aschwanden et al. 1999; Roberts 2000; De Moortel et al. 2002b) or simultaneously in the $171 \AA$ TRACE bandpass and the $195 \AA$ bandpass using SoHO/EIT (Robbrecht et al. 2001). De Moortel et al. (2002a,c) studied 38 TRACE examples of oscillations and found that most of the EUV propagating disturbances propagate outwards from the footpoints of large diffuse coronal loops

Send offprint requests to: A. De Groof, e-mail: Anik.DeGroof@wis.kuleuven.ac.be at an almost constant speed of $120 \pm 40 \mathrm{~km} \mathrm{~s}^{-1}$. The amplitude of the emission intensity perturbations is usually less than $6 \%$ of the background. The characteristic periods are several hundred seconds (145-525 s) and the remarkable presence of 3-min and 5-min oscillations points strongly in the direction of an underlying driver exciting the loop footpoints. Typically, the propagating intensity variations damp very quickly - they are only observed within the first $20 \mathrm{Mm}$ along the loop - and no manifestations of downward motions are found. Nakariakov et al. (2000) and Tsiklauri \& Nakariakov (2001) developed a theoretical model interpreting these periodic intensity oscillations as damped slow magneto-acoustic waves in a stratified atmosphere.

A totally different context in which intensity disturbances are observed are flare loops: propagating intensity variations are then attributed to plasma material falling down along the magnetic legs of a coronal loop after the eruption of a solar flare. Most observations are taken in chromospheric lines like the Ca II-line and $\mathrm{H} \alpha$ (e.g. Loughhead \& Bray 1984; Delone et al. 1989; Heinzel et al. 1992; Wiik et al. 1996). Since the loops are usually only partly filled with $\mathrm{H} \alpha$ - or Ca II-emitting material, the fine structure revealed by high spatial resolution 
observations consists of bright blobs moving downwards which are often almost equidistant leading to terms like "train of blobs". In some, more exceptional, cases a continuous motion from one footpoint to the other is observed.

Another possible origin of downflowing material is a prominence. In this case too, cool material drains down along magnetic field lines and since the plasma density and temperature are never homogeneous, the cool plasma is seen as falling bright blobs in cool chromospheric lines like $\mathrm{H} \alpha$ and $\mathrm{Ca}$ II (Engvold et al. 1979).

In this paper, to the best of our knowledge, the first report is given on propagating disturbances in the EUV band $304 \AA$, a wavelength band observed by e.g. the Extreme-ultraviolet Imaging Telescope, EIT (see Delaboudinière et al. 1995). The dominant part of the plasma emitting in the EIT $304 \AA$ band has temperatures from 60000 to $80000 \mathrm{~K}$. As a consequence both plasma cooling down after a flare or draining from a cool prominence is visible in EIT $304 \AA$, as well as waves and instabilities propagating in plasma at these temperatures. Therefore, it is not that easy to understand the nature of intensity variations in this EUV band when no clear or evident cause is noticable nearby. The intensity variations studied in this paper are of this kind. Since the time cadence of the EIT $304 \AA$ instrument is limited in nominal mode, we use the so-called EIT shutterless mode with a high time cadence in order to be able to see intensity variations with periods of the order of a minute.

In the next section, we first describe the technical details of the EIT shutterless mode and the calibration which is needed before the images can be analyzed. We will concentrate on one particular dataset: the sequence of 11 July 2001. The solar features visible in this sequence are described in Sect. 3. A multiwavelength study is presented in order to get more insight in the connections or possible causal links between the phenomena seen in the data. In Sect. 4, the intensity variations are analyzed in detail and the characteristics are summed up. Finally, in Sect. 5, we discuss the nature of the propagating disturbances and the arguments to interpret them as a wave or flow. At the end of the paper we give a summary and a discussion of the results.

\section{EIT shutterless program}

The data analyzed in this paper are obtained during a so-called "EIT shutterless campaign". This program in which EIT takes a high-cadence sequence of images without closing the shutter, was started in December 2000 by the Royal Observatory of Belgium (Clette 2000). Every 3 months, a sequence of 120 images is taken with a time cadence of $68 \mathrm{~s}$, interrupted only by two gaps allowing each for a LASCO C2 image in order to minimize the disturbance of the EIT "CME watch program". The series of campaigns aims to monitor long-term changes in the small-scale dynamics of all types of solar structures: active regions, quiet sun, coronal holes, etc. In order to monitor the transition region as well as the corona, the sequences are taken in two bandpasses, either $304 \AA$ or $195 \AA$. This changes from campaign to campaign. Instead of the normal full disk field of view $($ FOV) which is $1024 \times 1024$ pixels large, the EIT shutterless program concentrates on a subfield of $416 \times 416$ pixels ( 1 EIT pixel $\approx 2.6$ arcsecs). The FOV needs to include as

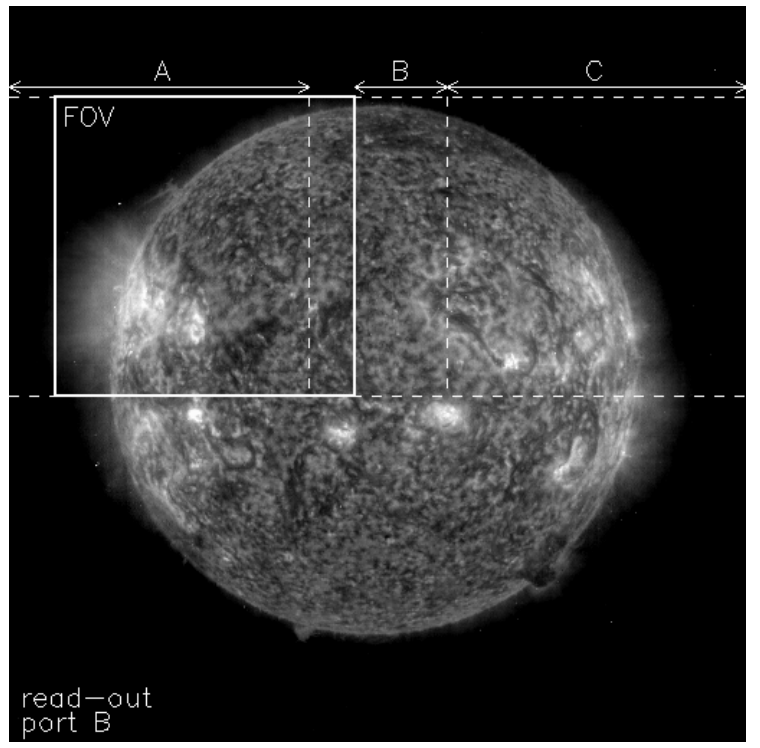

Fig. 1. The field of view (FOV) of the EIT shutterless sequence of 11 July 2001 (solid lines). The dashed lines indicate the regions A, $\mathrm{B}$ and $\mathrm{C}$ where the image information gets contaminated during read-out.

many (preferably all) types of solar regions as possible, including the solar limb, and therefore a quarter-disk is generally considered. Because of the importance of multi-wavelength studies, up till now the program has always been supported by TRACE (171 $⿱$ ) and, in addition, other instruments like CDS, MDI, SPIRIT (onboard the CORONAS-F satellite), etc. join the campaigns whenever possible.

As these EIT movies have a high cadence (68 s) and exposure time $(\approx 50 \mathrm{~s}$; see discussion later), features which are mainly buried in dynamics become visible and causal links which are unobservable in static images are revealed. On the other hand, keeping the shutter open during the whole sequence leads to unphysical smears throughout the image since the CCD pixels are read out while the shutter is still open so that photons falling in onto the CCD during the read-out process are still registered. Therefore an adapted calibration program is needed to clean the images (see Berghmans 1998). For the technical explanation of the calibration of these kind of shutterless sequences, we will describe the different calibration steps followed in the particular example of July 11, 2001. This shutterless mode sequence had the following setup (see Fig. 1):

- the FOV is a $416 \times 416$ subfield in the NE quadrant [65:480, 500:915] (EIT image pixel coordinates),

- read-out port $\mathrm{B}$ at the bottom left of the CCD,

- 120 images (304 $\AA$ ) between 16:00 UT and 18:28 UT with an average cadence of $68.2 \mathrm{~s}$,

- two gaps after the 40th and the 80th image for a LASCO C2 image.

Before and after the sequence, two full disk $1024 \times 1024$ images were taken in $304 \AA$ at 15:37 UT and 18:42 UT respectively.

The general procedure is as follows. After being exposed, a shutterless mode image of the subfield "FOV" (field of view) is transferred to the read-out port in the bottom left corner of 
the CCD. According to Delaboudinière et al. (1995), reading out a subfield takes three steps. Firstly, all 64 columns ahead of the FOV are dumped at a rate of $0.480 \mathrm{~ms}$ per column until the left edge of the FOV has shifted to the left edge of the $\mathrm{CCD}$ and the charges collected in region FOV move to region $\mathrm{A}$ (see Fig. 1). Since the shutter remains open, the FOV image is contaminated by photons collected during the transfer through regions $\mathrm{A}$ and the remaining transfer path in the FOV itself. In a second step the actual read-out of the FOV image (now present in region A) takes place at a rate of $20.8 \mathrm{~ms}$ per column. Note that an additional $0.48 \mathrm{~ms}$ per column is needed since the pixels also have to be dumped after read-out. Furthermore, during this reading process the pixels in the FOV get further contaminated in region A: the more to the right in the FOV, the more contamination. For every column which is read out, a "black" column appears at the right hand side of the CCD (region C) which then further drifts leftwards when new columns are read out. In this process a smeared-out image of the far western side of the Sun is collected in region C. As a final step in the process, the remaining 1024-64-416=544 columns following the chosen subfield are dumped, again at a rate of $0.48 \mathrm{~ms}$ per column. Note that in this process the smear-out image that was collected in region $\mathrm{C}$ is moved in the FOV. Moreover this smear-out image of the far western side of the Sun is contaminated during the transfers through region B and the FOV. It is important to note that, since we do not do any "CCD clears" during the sequence, this contaminated smear-out image remains in the FOV when the exposure of the next image starts.

So, to summarize, a subfield "image" consists of the following contributions:

- smear-out of region $\mathrm{C}$ with an effective exposure time of $t_{1}=416 \times 20.8 \mathrm{~ms}=8.6528 \mathrm{~s}$;

- smear-out of the transfer through region B and the FOV with an effective exposure time of

$t_{2}=(544-1) \times 0.48 \mathrm{~ms}=0.2606 \mathrm{~s}$;

- true solar image in the FOV region with an at this stage unknown exposure time of $t_{3} \mathrm{~s}$;

- smear-out of the transfer through the FOV and region A until the left edge of the FOV reaches the left edge of the CCD: $t_{4}=(64-1) \times 0.48 \mathrm{~ms}=0.03024 \mathrm{~s}$;

- smear-out of region A during the reading-out and dumping process: $t_{5}=416 \times(20.8+0.48) \mathrm{ms}=8.8525 \mathrm{~s}$.

Since there are no CCD clears, the total exposure time $t_{1}+t_{2}+$ $t_{3}+t_{4}+t_{5}$ must equal the time interval between the observation of the preceding image and the present image. This allows us to derive the effective exposure time $t_{3}$ of the true solar image in the FOV:

$$
\begin{aligned}
t_{3} & =\text { obstime }(i)-\text { obstime }(i-1)-t_{1}-t_{2}-t_{4}-t_{5} \\
& =\text { obstime }(i)-\text { obstime }(i-1)-17.7961 \mathrm{~s} \\
& \approx 68.2 \mathrm{~s}-17.8 \mathrm{~s} \approx 50.4 \mathrm{~s} .
\end{aligned}
$$

Let us now show what the combination of contaminations 1,2 , 4 and 5 looks like. In Fig. 2 (top) one frame of the shutterless sequence (at 16:02 UT) is plotted (left) together with the total smear which we estimated to be present in that image (right).

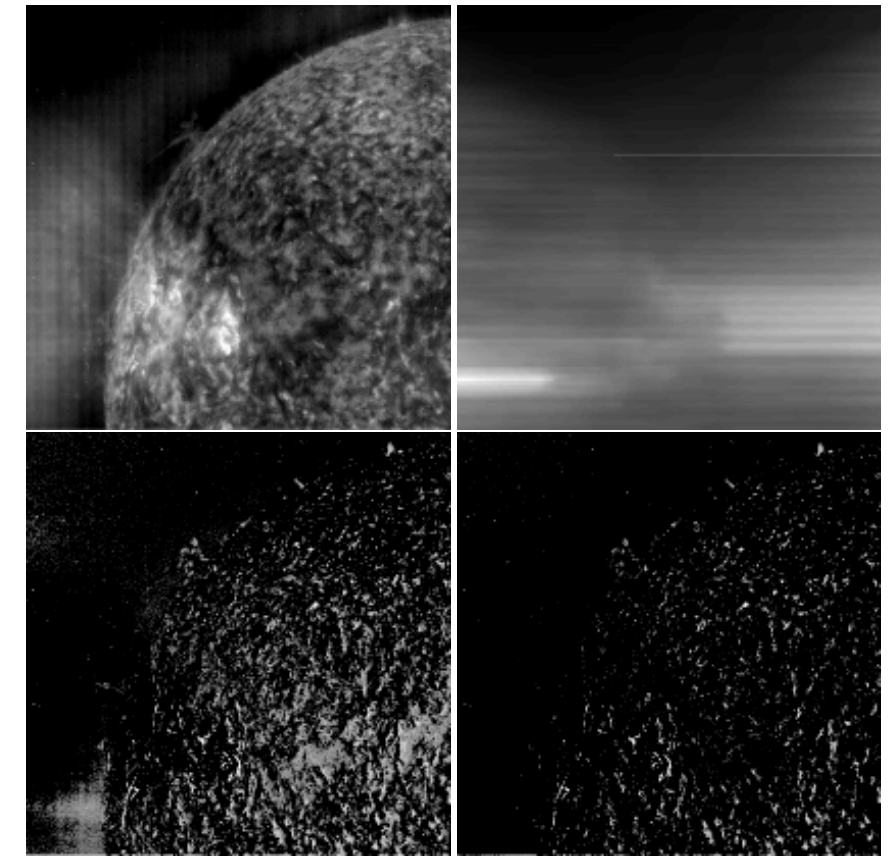

Fig. 2. Top left: 2nd frame of the shutterless sequence at 16:02 UT; Top right: total smear estimated using the full disk image at 15:37 UT; Bottom left: subfield of the full disk image subtracted from the 2nd frame of the shutterless sequence; Bottom right: bottom left image minus estimated smear. Note that different intensity scales are used for each image for optimal contrast.

In order to calculate this smear, we used the nearby full disk image at 15:37 UT. We integrated over the appropriate columns of this full disk image to calculate the 4 different contaminations and using the above deduced "effective exposures" for each contamination, we summed up the 4 contaminations which resulted in Fig. 2 (top right). In order to check this estimate, the bottom left image in Fig. 2 shows the difference between the corresponding subfield of the full disk image, taken in nominal mode, and the frame of the shutterless sequence. When comparing the top right image with the bottom left one, similarities are seen in the brighter and darker regions of the image. In order to make the similarities more clear, we subtract the top right image (estimated smear) from the bottom left image (sequence image minus subfield full disk image) and get the result in the bottom right part of Fig. 2. The features which are still visible are due to the solar rotation between 15:37 and 16:02 UT, which is why all features are bright on the right (later times) and dark on the left (earlier times), and due to the solar activity changes during this time interval. With the above method of smear estimation, it is not possible to take into account the activity changes but the solar rotation we can correct for.

Apart from the smear, the sequence should also be corrected for contaminations like cosmic rays, missing blocks, flat field emission, traces of the grid, etc., and additional calibration techniques like exposure time normalization, filter factor correction and normal response function should be applied. Since the standard "EIT_PREP"-program cannot be applied on shutterless sequences, we use similar IDL techniques, implemented directly into the code we designed to read, calibrate and 


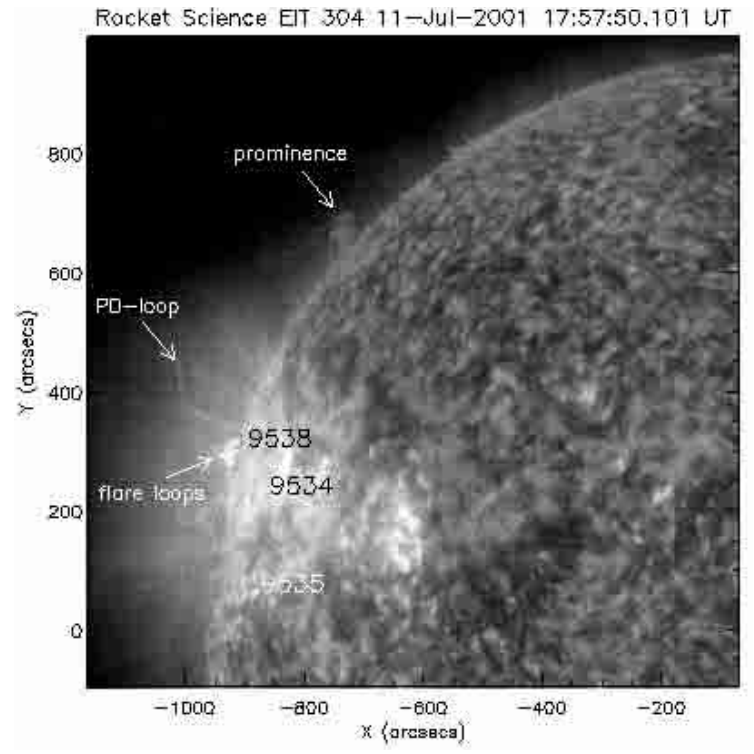

Fig. 3. One of the frames in the EIT $304 \AA$ shutterless sequence.

analyze the series of images. Only a correction for cosmic rays is not applied as we experienced an important loss of information in the fine structure of the images. As we aim to investigate small scale intensity variations, these details are too important for the analysis. The software we used is still in the experimental phase but will be made a public part of the solar soft library when more refinements are implemented.

\section{Observations}

As shown in Figs. 1 and 3, the field of view of the $304 \AA$ sequence under analysis is the north-east quarter disk, shifted one block (i.e. a data cube of $32 \times 32$ pixels) to the east and one block to the south. The EIT-campaign started at 16:00 UT and lasted until 18:28 UT during which time 120 full-resolution images $(416 \times 416$ pixels $)$ were taken with a $68 \mathrm{~s}$ time cadence. Looking at the presence of typical solar regions and features, most types are present: near the east limb some large and complex active regions (NOAA 9534, 9535 and 9538) are visible, and close to the north pole, the field of view includes a part of the polar coronal hole (especially visible in higher temperature bands). In addition, a large quiescent prominence is crossing the north east limb towards the active region NOAA 9534. Part of the prominence is visible in emission over the north-east limb, the rest is showing up as a dark filament on the solar disk, continuing south in between NOAA 9538 and 9534 (see Fig. 3).

Running the sequence as a movie, several interesting dynamic features become visible ${ }^{1}$. First of all, an off-limb loop structure above active region NOAA 9538 shows intensity variations propagating downward during the whole sequence (in the remainder of this paper, we will abbreviate these propagating disturbances as PDs). South of this loop, a smaller and more complex off-limb loop structure expands and brightens

\footnotetext{
${ }^{1}$ An EIT movie is provided on the SoHO "Hot shots"-website http://sohowww. nascom.nasa.gov/hotshots/2001_07_16. In addition, a part of the corresponding TRACE movie is made available.
}

Table 1. General characteristics of the data available in the time frame of the EIT shutterless on 11 July 2001.

\begin{tabular}{llcccc}
\hline \hline Instrument & Bandpass & Start & End & Cadence & \# Images \\
\hline EIT & He II $(304 \AA)$ & $16: 00$ & $18: 28$ & $\sim 68 \mathrm{~s}$ & 120 \\
TRACE & Fe IX/X (171 ̊) & $16: 00$ & $18: 20$ & $30 \mathrm{~s}$ & 227 \\
SXT & Soft X-ray & $15: 10$ & $16: 12$ & irregular & 33 \\
& Soft X-ray & $16: 46$ & $17: 50$ & irregular & 33 \\
& Soft X-ray & $18: 25$ & $19: 20$ & irregular & 14 \\
Big Bear & H $\alpha$ & $15: 43$ & $00: 33$ & $30 \mathrm{~s}$ & 1060 \\
EIT & Fe IX/X $(171 \AA)$ & $01: 00$ & $19: 16$ & $\sim 6 \mathrm{~h}$ & 4 \\
EIT & Fe XII $(195 \AA)$ & $00: 00$ & $23: 48$ & $12 \mathrm{~min}$ & 111 \\
EIT & Fe XV $(284 \AA)$ & $01: 06$ & $19: 22$ & $\sim 6 \mathrm{~h}$ & 4 \\
\hline
\end{tabular}

up while north-west of these two phenomena, the dark filament shows intensity variations moving northward along the spine of the filament connecting it to the part of the prominence seen off-limb. In addition, the east and north limb show several (macro) spicules and plasma flows along magnetic loops.

Given its peculiarity and its prominent presence in the sequence, our main interest is in the loop system with the downward propagating intensity variations. As described in the Introduction, such intensity variations are often observed in $\mathrm{H} \alpha$, propagating downward along cooled-off flare loops. Therefore we first investigate the available data in other wavelengths to see whether the PDs are also flare related (see Table 1 for all the data available in the EIT timeframe). According to GOES, indeed a C2.4 flare went of in the time interval of the observation with its peak occurring at 17:11 UT. An analysis of Yohkoh-SXT data confirmed that this flare was included in the field of view under analysis. However, there seems to be no clear relation between the flare and the downward motions along the PD-track. Figure 4 shows an SXT image at 17:48 UT, overplotted by the contours of EIT $304 \AA$ at the same time. The low-lying bright and expanding off-limb loops in the EIT-sequence appear at exactly the same location as the SXT flare and hence, they are most probably (post)flare loops cooling down. On the other hand, the long contour, north of the flare site, which is coloured white corresponds with the PD-track in EIT. Throughout the whole SXT sequence (ranging from 15:10 until 19:20 UT), there is no sign of this loop nor the propagating intensity variations. We also checked the other data available in higher temperatures than $304 \AA$ but both TRACE $171 \AA$ and the EIT wavelengths $171 \AA, 195 \AA$ and $284 \AA$ do not show any sign of the PD-track nor the intensity variations. Only in a TRACE $171 \AA$ movie where from each image an average image is subtracted, a slight downward motion is seen, which looks fairly continuous nevertheless. The flare loops south of the PD-track on the other hand are observed in great detail.

In conclusion, since it has no hot counterpart and it does not have the typical long duration characteristics of a flare loop (it remains at the same altitude), the PD loop system is most probably not a cooling (post)flare loop. Moreover, the intensity 


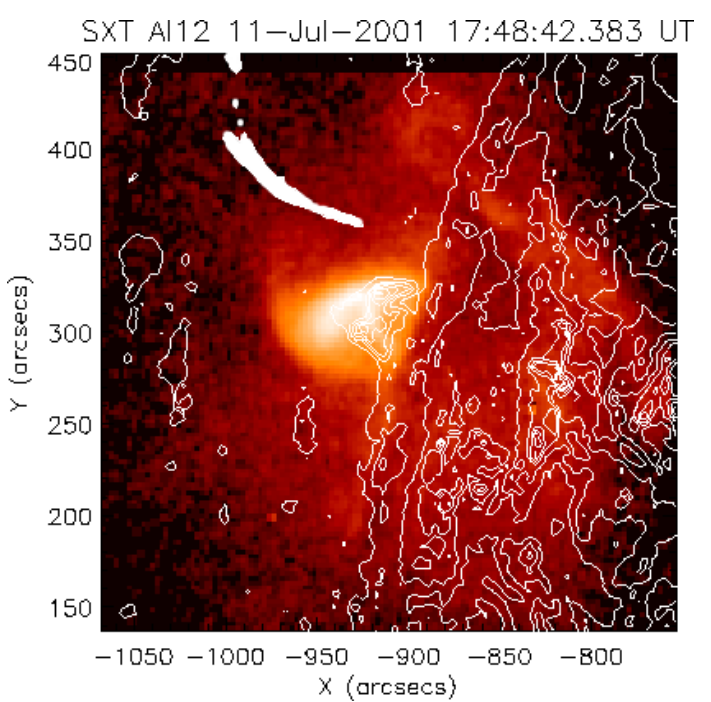

Fig. 4. SXT image of the flare region at 17:48:42, overplotted by the contours of EIT $304 \AA$ at 17:48:44. The contour, north of the flare site, which is coloured white corresponds with the downward motion in EIT.

variations are observed before the flare even went off and therefore we conclude that these two phenomena are not closely related.

Is it visible in lower temperatures then? Since the signal in the EIT $304 \AA$ bandpass is dominated by He II emission of plasma around $80000 \mathrm{~K}$, we hope to see similar plasma features in the $\mathrm{H} \alpha$ line which emits at chromospheric temperatures $(20000 \mathrm{~K})$. We checked $\mathrm{H} \alpha$ images from the Big Bear Solar Observatory in California and indeed found bright signatures of the loop's footpoint. Moreover, some bright dots higher up perfectly correspond to the downward moving blobs in EIT. A more detailed study of the $\mathrm{H} \alpha$ data is ongoing and the results will be presented in a future paper. Nevertheless, from these preliminary results we can already conclude that the bright "blobs" seen in EIT $304 \AA$ are collections of cool plasma (20 000-80000 K) in hotter surroundings ( 1 MK).

\section{Detailed analysis of intensity variations}

In order to analyze the downward motions in further detail, we follow the method used by Wiik et al. (1996) in their analysis of intensity variations along off-limb loops in $\mathrm{H} \alpha$. The loops in Wiik et al. are definitely related to a flare, i.e. they were cooled-off flare loops which co-existed with hot loops, while our PD-track has no hot loop counterpart. Nevertheless, the bright plasma blobs they observed moving downward along the flare loops look very similar to the intensity variations we observe in the EIT shutterless sequence. In addition, the height of the PD-track, approximately equal to $10^{5} \mathrm{~km}$, exactly corresponds to the height of the flare loops in the Wiik analysis. Therefore we follow the same method.

Firstly we select different "blobs" of enhanced intensity in order to follow them in time throughout the whole sequence. Secondly, we outline the PD-track and make a location-time plot along it. In such an image, an intensity disturbance propagating along the analyzed structure can be identified as clear

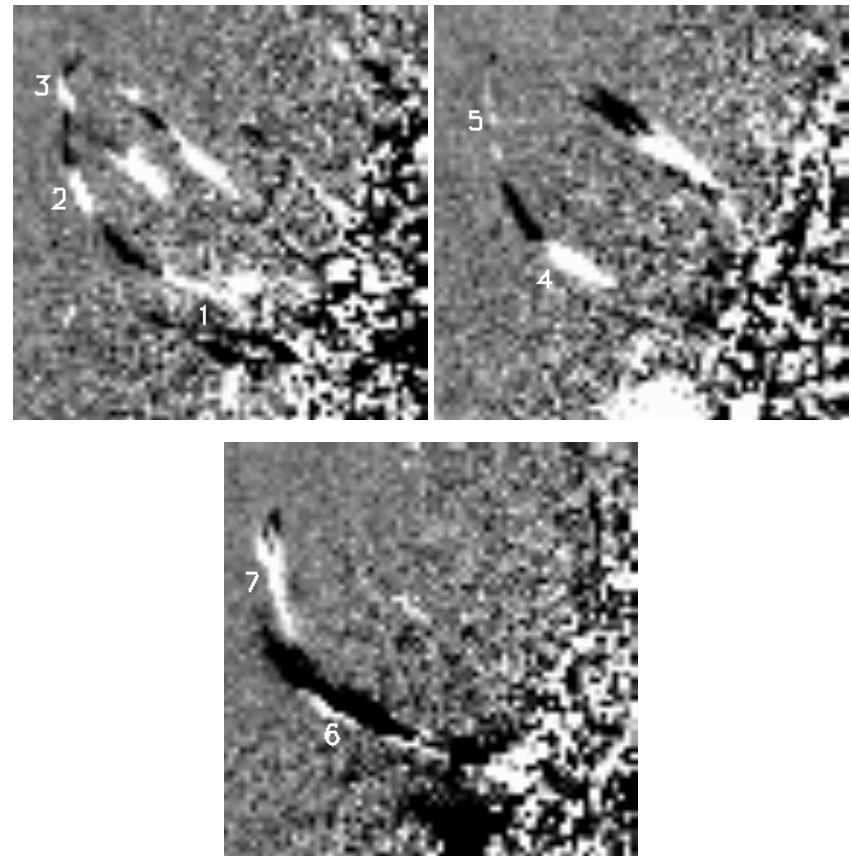

Fig. 5. Three difference images taken at 16:27, at 17:09 and at 17:58, allowing for the identification of the 7 blobs moving down along the PD-track. The additional blobs propagating along straight paths move with comparable speeds and are not discussed in this paper.

diagonal bright bands. In a third step, the evolution of the identified "blobs" can be followed and the speeds can be calculated by measuring the slope of the bright ridges. In Sect. 4.2, we measure the apparent amplitudes of the intensity variations to learn more about their nature. This information, together with the comparison of the measured blob velocities with characteristic wave speeds and with the free-fall speed, then helps to interpret the motions as waves or flows in Sect. 5.

\subsection{Identification of "blobs" and evolution along track}

By means of difference images (from each frame we subtract the frame taken roughly 5 min earlier), the contrast of the images in the sequence can be enhanced and the different bright "blobs" can easily be identified in 3 images taken at 3 different times in the sequence (see Fig. 5). In the observed time frame we find 7 blobs which are formed close to the top and all propagate down along the curved track of the off-limb loop.

In the next step we outline the track most of the blobs are following (see Fig. 6a) and collect the intensity information of each pixel along the track and the pixels in its direct neighbourhood for each frame in the sequence. All counts in a box of 5 by 5 pixels around each track pixel (spanning 13 arcsec) are summed together and used to construct the intensity profile along the track at time $t$. The set of these profiles for each moment in time are combined in the location-time plot in Fig. 6 b. The columns in the plot show the intensity profiles along the track and time is plotted on the horizontal axis.

Clear bright ridges with a negative slope show up in the plot, corresponding to the 7 "blobs" which move down along the track. (In the bottom left part of the image, an extra bright 

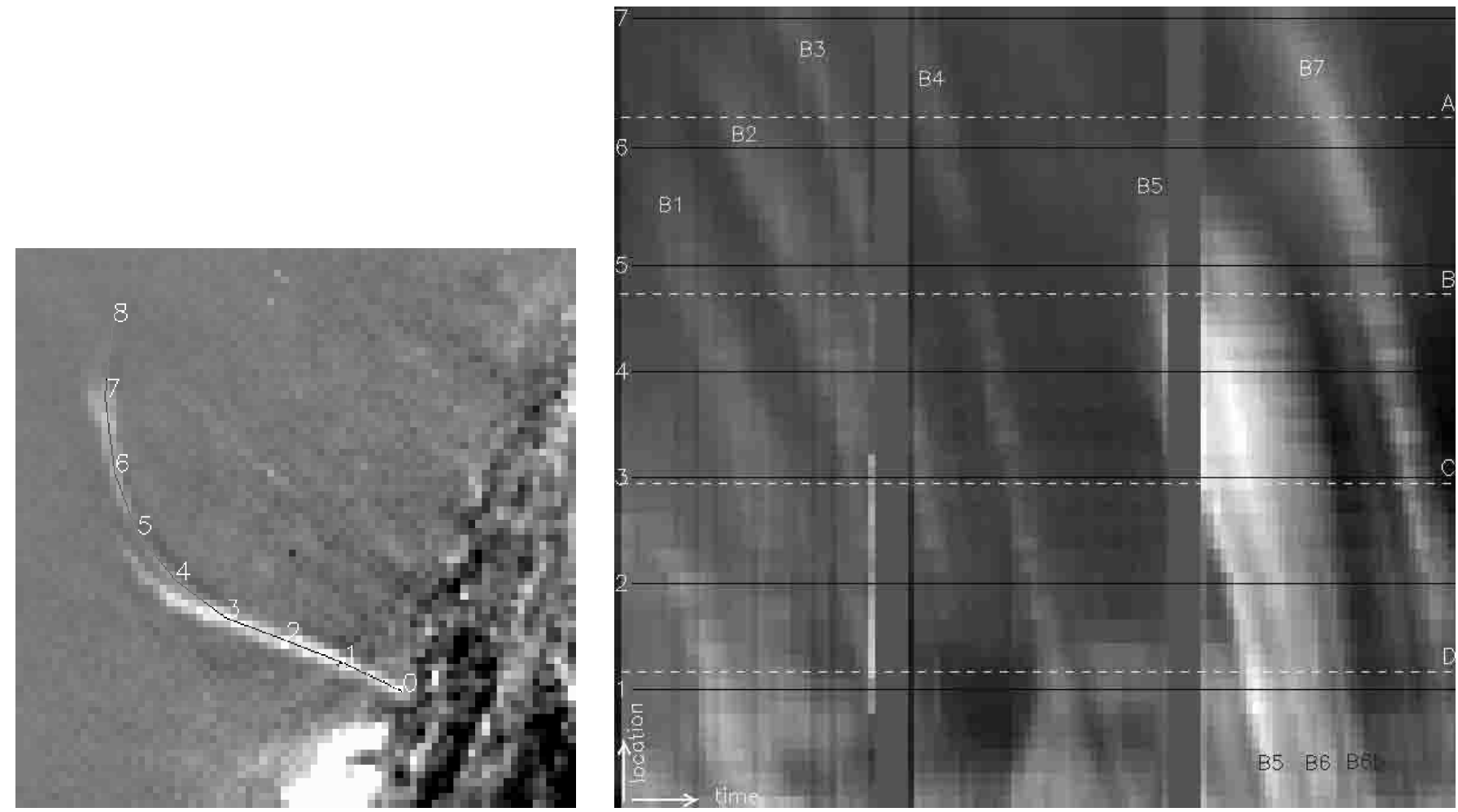

Fig. 6. Left: the track of the intensity variations, outlined in 1 frame of the EIT-sequence (at 17:58). Right: location-time plot of the outlined track. The location numbers in the left figure correspond to the numbered black lines on the right. The ridges due to the blobs passing by are labelled B1 to B7.

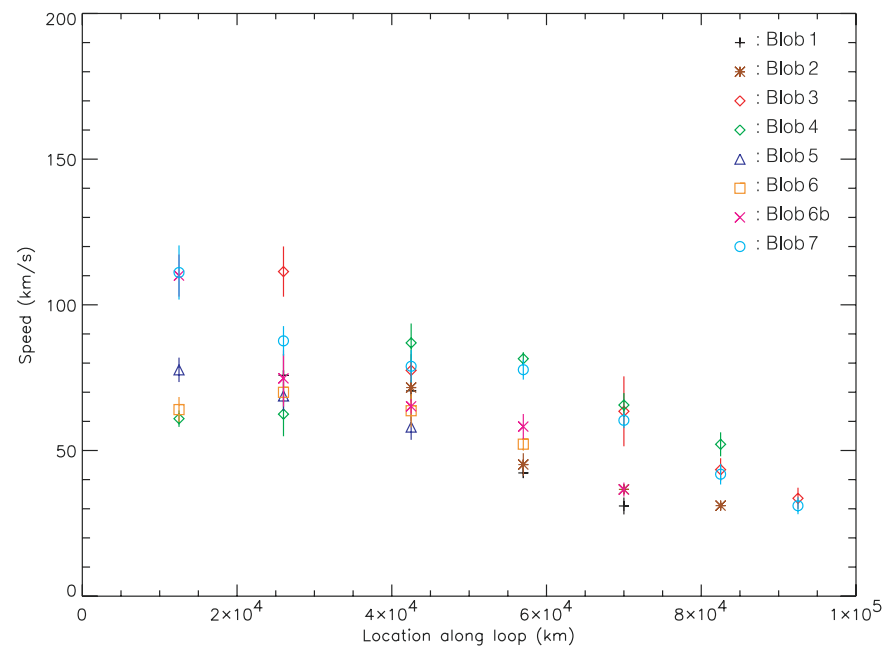

Fig. 7. The measured speeds of each "blob" at several locations along the loop.

ridge shows up which could be labelled "B0" but since it was already on its way down when the sequence started, we do not include it in our analysis.) For blob $6-$ "B6" in the figure we also measured the slope resulting from the sharp end of the bright blob passing by, we called it "B6b". The fact that the bright ridges are not straight but curved shows that the velocities of the different blobs are not constant but increasing, since the slopes of the ridges become steeper closer to the limb. All blobs seem to follow this evolution. By measuring the slopes of each ridge at several locations along the loop (marked by the numbers in Fig. 6a and the black horizontal lines in Fig. 6b), we can calculate the apparent speed of the intensity variations at each location. We write "apparent" since, taking into account the line-of-sight projection effects, the measured values are only a lower limit for the true propagation speeds.

All the measured speed values are combined in Fig. 7. The locations 1 to 7 along the loop are translated to their projected heights above the limb and at each location, the mean of several measurements of the ridge's local slope (4 to 8 for each datapoint) is plotted. The error bars show the standard deviation of the results found by fitting the bright ridge locally several times with a straight line. As shown in the figure, all datapoints form a compact cloud of speeds increasing from roughly $40 \mathrm{~km} \mathrm{~s}^{-1}$ a the top of the loop $(100 \mathrm{Mm})$ to about $120 \mathrm{~km} \mathrm{~s}^{-1}$ near the limb. Before we proceed with the interpretation of the measured speeds, we first calculate the effect in the loop's intensity when blobs pass by.

\subsection{Amplitudes of the intensity variations}

In order to calculate the amplitudes of the propagating intensity variations, we spot different locations along the PD-track and study the intensity evolution of these particular locations in time. In practice, this idea corresponds to the study of the profiles found through a horizontal cut in the location-time plot of Fig. 6b. We arbitrarily choose 8 different positions along the loop, roughly equidistant along the total height. At each position, we plot the intensity profile in time in order to measure the intensity enhancement associated which each "blob" passing by. In Fig. 8, we show the profiles at heights A, B, C, 

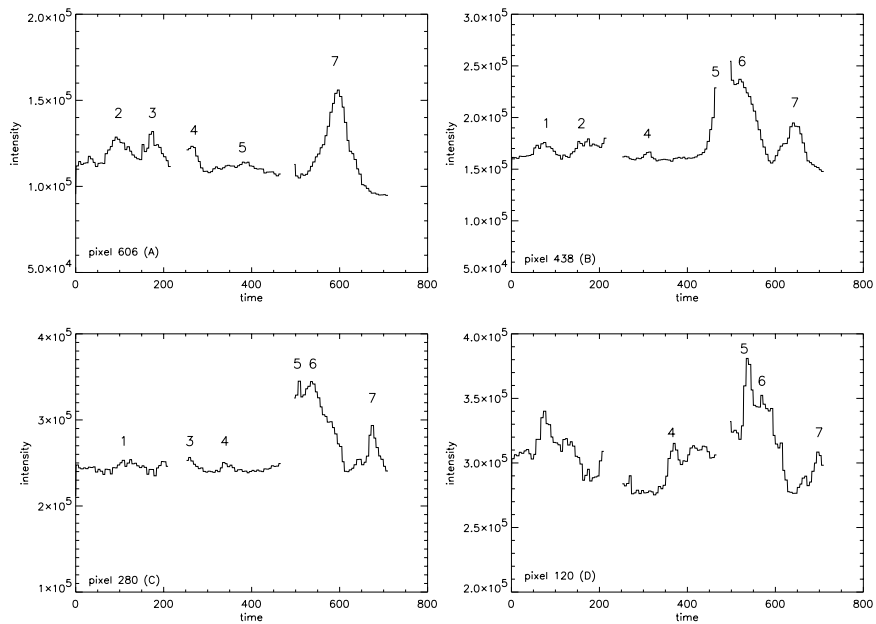

Fig. 8. The intensity evolution (in DN) in time at 4 positions along the track: at positions A, B, C and D (see Fig. 6b). The two intervals of missing data in each plot correspond to the two data gaps in the sequence.

Table 2. The relative intensity enhancement $\mathrm{d} I / I$ of each of the 7 blobs (columns) as a percentage of the background intensity. The intensity enhancements are calculated at 8 different positions along the track (rows).

\begin{tabular}{l|ccccccc}
\hline \hline Position (pixels) & B1 & B2 & B3 & B4 & B5 & B6 & B7 \\
\hline $606(A)$ & $/$ & 15 & 18 & 17 & 6 & $/$ & 48.6 \\
514 & 8.7 & 19 & 10.8 & 12.7 & $(17.5)$ & $(22.4)$ & 42 \\
$438(\mathrm{~B})$ & 10 & 12 & $/$ & 5.7 & $/$ & 51.9 & 31 \\
361 & 13 & 19 & $/$ & 13.4 & $/$ & 69.1 & 43 \\
$280(\mathrm{C})$ & 8 & $/$ & 6.7 & 4.6 & 44.4 & 43.8 & 24 \\
208 & 6 & $/$ & 8.3 & 9.5 & 36.2 & 33.1 & 22 \\
$120(\mathrm{D})$ & $/$ & $/$ & 5.5 & 14.5 & 38 & 27.5 & 14 \\
45 & $/$ & $/$ & $/$ & 15 & 24.5 & 17.3 & 6 \\
\hline mean & 9.1 & 16.3 & 9.9 & 11.6 & 27.8 & 37.9 & 28.8 \\
st.dev & 2.6 & 3.4 & 5.0 & 4.5 & 14.4 & 18.3 & 15.0 \\
\hline d $n / n$ & 4.6 & 8.1 & 4.9 & 5.8 & 13.9 & 18.9 & 14.4 \\
\hline
\end{tabular}

and $\mathrm{D}$, indicated in Fig. $6 \mathrm{~b}$ as dashed lines and corresponding with pixels $606,438,280$, and 120 along the track where pixel 0 is located at the limb and pixel 680 near the top of the loop. Remark the different scales of the vertical axes in the four plots of Fig. 8. The numbers of the different blobs passing by are indicated in the plots. The two intervals of missing data in each plot correspond to the two data gaps in the observational sequence, after the 40th and 80th image respectively.

The amplitudes associated to each blob at each location are measured by calculating the relative intensity enhancement with respect to the background intensity, which is defined by the minimum intensity in between two blobs passing by. The calculated values are summarized in Table 2. Missing values are due to the data gaps or to an unclearly defined ridge. For
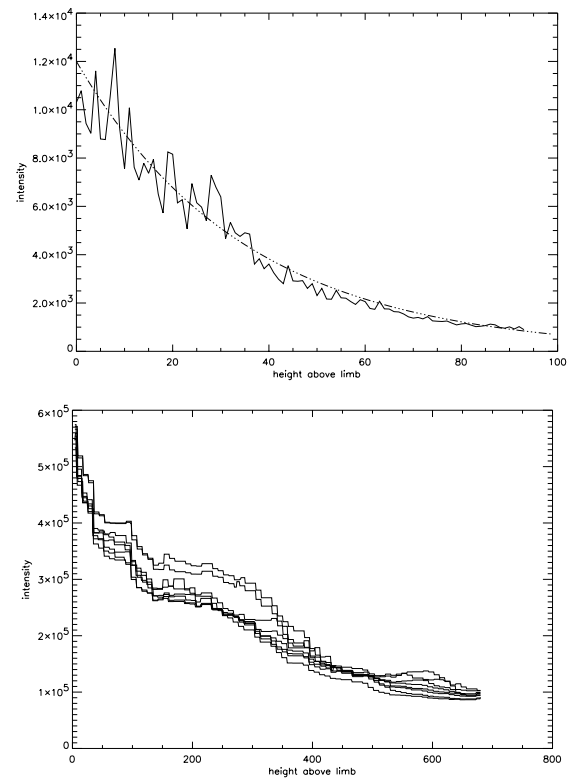

Fig. 9. Top: The intensity profile of a quiet region off-limb from the limb to a height of approximately $10^{5} \mathrm{~km}$, fitted by the exponential function $12000 \exp (-x / 35)$. Bottom: The intensity profiles along the PD-track (from limb to top) at different moments in time.

the amplitudes of blob 5, the minimal intensity before blob 5 or after blob 6 is taken as background since there is not enough interblob space between blobs 5 and 6 to give a reasonable value. The mean and standard deviation of the relative intensity enhancement $\mathrm{d} I / I$ for each blob is indicated at the bottom of the table. To get an idea of the relative density enhancement $\mathrm{d} n / n$ which is related to this, the value for $\mathrm{d} I / I$ divided by two is a good approximation since $I \propto n^{2}$.

We remark that the percentages are strongly influenced by the change in background intensity when moving closer to the limb. As it is clear in Fig. 9a, the profile of the intensity along an arbitrary radial cut starting from the solar limb and moving outwards, can be fitted by an exponential function. Therefore, as the background intensity increases towards the limb, the relative intensity enhancement of the "blobs" decreases. This evolution is indeed visible in Table 2 and leads to the relatively high standard deviations at the bottom of the table. Far from the limb, the relative intensity enhancement is substantially larger than close to the limb, except for cases where the blob has not really formed yet close to the loop top. Hence a logical next step would be to subtract an exponential fit of the absolute minimum intensities at all locations; these absolute minima correspond best with the "background intensity" of the loop. However, since all profiles along the loop (plotted by taking several vertical cuts of Fig. 6b) show a systematic deviation of the exponential fit - they all show a bump halfway the loop (see Fig. 9b) - we chose to subtract at each location the absolute minimum intensity at that location, lowered by the absolute minimum value at location A. Thus, nothing is subtracted at the top of the loop, and the procedure only affects the smaller heights, especially those close to the limb. With this new method we get the relative intensity enhancements listed in Table 3. These new percentages are definitely higher than 
Table 3. The relative intensity enhancement $\mathrm{d} I / I$ of each of the 7 blobs (columns) as a percentage of the background intensity, after the subtraction of the background intensity (minimal intensity for each location). The intensity enhancements are calculated at 8 different positions along the track (rows).

\begin{tabular}{l|ccccccc}
\hline \hline Position (pixels) & B1 & B2 & B3 & B4 & B5 & B6 & B7 \\
\hline $606(\mathrm{~A})$ & $/$ & 35.8 & 39.0 & 29.5 & 20.0 & $/$ & 64.2 \\
514 & 17.4 & 31.1 & 23.8 & 12.2 & 28.0 & 44.8 & 51.1 \\
$438(\mathrm{~B})$ & 27.9 & 31.1 & $/$ & 18.4 & $/$ & 92.1 & 47.9 \\
361 & 30.3 & 40.9 & $/$ & 29.3 & $/$ & 141.9 & 77.7 \\
$280(\mathrm{C})$ & 19.9 & $/$ & 22.0 & 15.7 & 115.7 & 115.7 & 62.0 \\
208 & 18.6 & $/$ & 23.9 & 24.9 & 98.6 & 90.2 & 61.8 \\
$120(\mathrm{D})$ & $/$ & $/$ & 15.5 & 41.8 & 111.3 & 80.7 & 41.8 \\
45 & $/$ & $/$ & $/$ & 54.4 & 112.3 & 84.9 & 42.8 \\
\hline mean & 22.9 & 34.7 & 25.9 & 28.3 & 66.7 & 92.9 & 56.2 \\
st.dev & 5.9 & 4.7 & 11.6 & 14.1 & 9.4 & 30.2 & 12.4 \\
\hline $\mathrm{d} n / n$ & 11.5 & 17.4 & 13.0 & 14.2 & 33.4 & 46.5 & 28.1 \\
\hline
\end{tabular}

the previous ones, firstly because we subtracted limb brightening which implies that the relative enhancement is calculated with respect to lower background values. Secondly, for every location we chose the absolute minimum in time (corrected for edge effects close to the gaps) instead of the local minimum, used in Table 2 as the background intensity of the loop. This choice explains the difference between the two approaches for location A, where no limb brightening is extracted. On the other hand, the new amplitudes are better related to the brightness and impact of the blobs as seen in the location-time plot (Fig. 6b).

However, we need to put the values in Table 3 in perspective. Although the calculated values give quite a fair idea of the intensity enhancement associated with a blob passing by, the magnitude of the values is strongly dependent on the method of background subtraction, i.e. the removal of effects which are not related to the loop itself. In addition, all values are based on the intensity variations along the specified track visualized in Fig. 6a. As it is clear in this figure, not all blobs follow exactly the same track along the loop. The most deviating example is actually visible in Fig. 6a: blob 6, the elongated bright structure already reaching the limb, clearly starts at an "off-track" position and only follows the outlined track at later times. Hence the ridge of "B6" only appears at later times and a lower position in Fig. 6b. These deviations also influence the results in Tables 2 and 3.

\section{Interpretation of intensity variations and speeds}

As explained in the introduction, intensity variations in the solar atmosphere can be caused by several different mechanisms. Also, it is not obvious whether to categorize the disturbances as a wave or plasma flowing along the loop.
A first possible indication for waves is periodic behaviour of the disturbances. Figures $6 \mathrm{~b}$ and 8 show bright blobs passing by at quite regular times but we cannot call it a real periodicity, especially because some blobs seem to elongate when they move closer to the limb (see e.g. Fig. 6a). However, since (quasi-)periodicity cannot be excluded, we compare the (projected) speeds we found with characteristic wave speeds. Since most of the waves found in the chromosphere or corona are interpreted as slow magneto-acoustic waves (see Introduction), we first calculate the typical value of the sound speed in plasmas within the observed temperature range $6-8 \times 10^{4} \mathrm{~K}$. Assuming the plasma to be isothermal, we use the formula

$C_{\mathrm{s}}=152 \sqrt{T} \frac{\mathrm{m}}{\mathrm{s}}$

to find a typical sound speed of $C_{\mathrm{s}} \approx 40 \mathrm{~km} \mathrm{~s}^{-1}$. Most speed values measured in Sect. 4.1 are definitely larger than this estimate (see Fig. 7), especially taking into account that they are only a lower limit for the true propagation speeds. Therefore the wave speed would be rather Alfvénic. Unfortunately, since the Alfvén speed is highly dependent on the magnetic field and the density, both uncertain in this case, we are not able to calculate a reliable estimate for the characteristic wave speed $v_{\mathrm{A}}$; it could range from 30 up to $2800 \mathrm{~km} \mathrm{~s}^{-1}$ (with the density $n$ ranging from $10^{8}$ to $10^{10} \mathrm{~cm}^{-3}$ and $B=1-10 \mathrm{G}$ ). Nevertheless, since pure Alfvén waves do not cause density changes, it is rather unrealistic that they would result in the big intensity variations seen in $304 \AA$.

Another comparison we can make concerns the amplitude. De Moortel et al. (2002c) measured the amplitude of the TRACE intensity variations as a percentage of the average background intensity where the latter is calculated for each position along the loops as the average flux during the observed time frame. They got an average relative amplitude of $4.1 \pm 1.5 \%$ within the range $0.7-14.6 \%$. The amplitudes we measured in the previous section are given as a percentage of the minimum intensity since the profiles deviated too much from a sinusoidal function to take the average. Therefore it is not straightforward to compare our results, i.e. mean relative intensity enhancements of 23-93\%, with the amplitudes found by De Moortel et al. (2002c). However, even when the amplitudes would be calculated with respect to the minimum intensity, an average TRACE wave would only cause an intensity difference around $9 \%$. Since our values convincingly exceed this number, we are confident to conclude that the propagating disturbances in the EIT sequence have a substantially larger effect on the intensity than a typical coronal wave.

A third difference with the bulk of waves observed in the solar atmosphere is the time evolution of the speed. According to De Moortel et al. (2002c), most waves travel with constant speeds around $150 \mathrm{~km} \mathrm{~s}^{-1}$ which is the typical sound speed in the corona. In contrast, the intensity variations observed in the EIT sequence are propagating with increasing speeds downward along the loop. They are observed along a $100 \mathrm{Mm}$ high loop and do not show any sign of damping while the TRACEwaves all damp heavily so that they are only observed within the first $20 \mathrm{Mm}$ along the loops. A lack of visible damping could be caused by a decrease of the loop's cross-sectional 


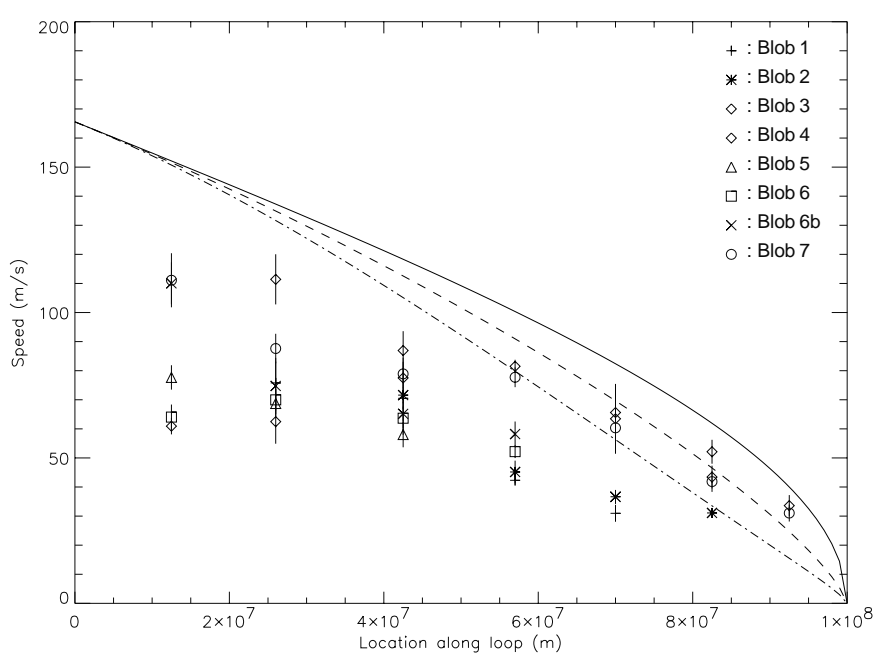

Fig. 10. The measured speeds of each "blob" at several locations along the loop, together with the theoretical free-fall speed along a vertical structure (solid line), along a semi-circular loop (dashed line) and along a semi-circular loop which is tilted $\pi / 4$ away from us (dotteddashed line). All curves assume a loop height of $100 \mathrm{Mm}$.

area from top to bottom but recent observations show that an average loop's cross-section is fairly constant (Klimchuk 2000) and only shows an important narrowing close to the footpoints (De Moortel et al. 2002c). And a last but very striking difference: slow magneto-acoustic waves are only observed propagating upwards while the bright blobs we see all move downwards!

These last arguments all point into the direction of plasma blobs flowing/falling down along the magnetic field lines. The gravitational acceleration would explain the increasing speed of the bright blobs and the fact that we only observe variations propagating downwards. In order to check this hypothesis we compare the speed values found above with the free-fall speed from a height of $100 \mathrm{Mm}$. In Fig. 10, the solid curve represents the free-fall speed along a vertical structure, i.e. perpendicular to the limb, with a height of $100 \mathrm{Mm}$. The measured speed values seem to follow this curve quite reasonably, especially close to the loop top. The fact that they are all smaller than the theoretical speed could be caused by the projection effect, although the deviation becomes larger when moving closer to the limb. Since we did not take into account the curvature of the loop, we plotted a second theoretical curve in Fig. 10 which represents the free-fall speed along a semi-circular loop (dashed line). As we are not sure about the possible projection angle, the dotteddashed curve assumes an angle of $\pi / 4$ between the loop plane and the plane of the sky, i.e. the plane of the loop is still perpendicular to the solar surface but tilted with respect to the plane of the sky. The choice of the angle $\pi / 4$ is rather arbitrary but not unrealistic seen the curvature and structure of the loop. Larger angles would lower the curve even more, smaller angles would more closely approximate the dashed curve. (We remark that a projection angle different from zero would cause the constant speed of e.g. a wave along a curved track to be observed as an increasing speed but the projection angle would have to be close to $\pi / 2$ to get a reasonable fit of the data and since we still observe the loop from aside, this is rather unrealistic.)

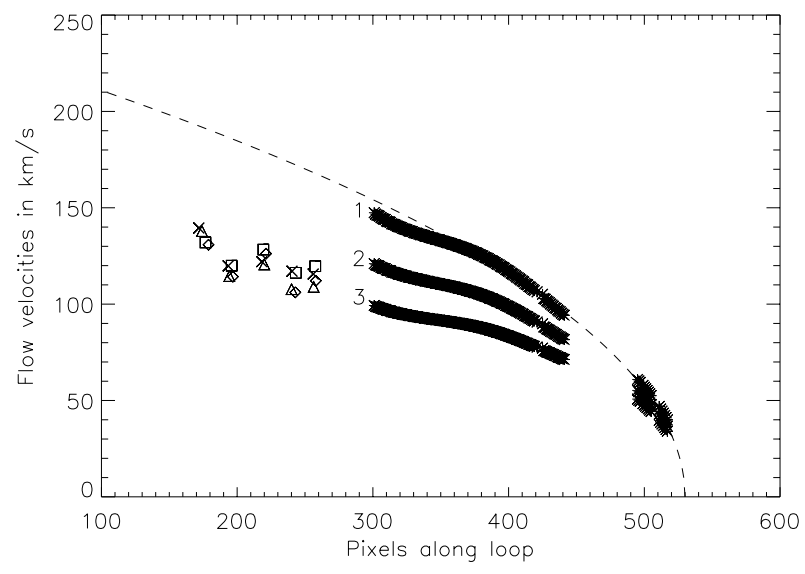

Fig. 11. Wiik et al. (1996): flow velocities along a flare loop leg of $100 \mathrm{Mm}$. The asterisks in the plot are computed from Doppler velocities using three slightly different shape models of the loop; the other symbols represent the velocities derived from the displacements of the blobs. The dashed line represents the free-fall velocity from a height of $100 \mathrm{Mm}$ (with kind permission of Kluwer Academic Publishers).

Since several parameters are uncertain, e.g. the exact height of the loop, the exact curvature (in addition deformed by helicity) and the projection angle, we could play around with these curves in order to fit the data more closely. Even so, for each plotted curve in Fig. 10, there is a strong deviation from the free-fall speed close to the limb, which suggests that the blobs are decelerated by some extra process.

This effect is seen earlier in almost all speed analyses of plasma blobs coming down along solar flare loops (Wiik et al. 1996; Heinzel et al. 1992; Loughhead \& Bray 1984), see e.g. the results found by Wiik et al. (1996) in Fig. 11. The height of the flare loop they investigated also equals $100 \mathrm{Mm}$ and the dashed line represents the free-fall velocity from such height. The asterisks in the plot are computed from Doppler velocities using three slightly different models reconstructing the shape of the loop from the wide range of datasets available; the other symbols represent the velocities derived from the displacements of the blobs. It is clear from the plot that they found a very similar departure from the free-fall velocity in the lower part of the loop. As reviewed by Wiik et al. (1996), several explanations for such plasma deceleration are proposed by different authors. Since for flare loops the gas pressure gradients of the cool gas seem to be insufficient to decelerate fast moving plasma (Heinzel et al. 1992), Loughhead \& Bray (1984) and Heinzel et al. (1992) suggested three other possible explanations: a global variation of the loop's magnetic structure during the typical fall-time of the blobs, deceleration by a presumed helicity of the magnetic field inside the loops, and deceleration of cool blobs as they move inside a hotter plasma. Mein et al. (1996) added an extra possible explanation namely a deceleration by shock waves generated at the footpoints of the loops, presumably due to the interaction of downflows with denser parts of the atmosphere. The comparison with prominences can also be made since prominence plasma is supported against gravity by Alfvén waves (Jensen 1990). As discussed 
by Wiik et al. (1996), some of these mechanisms were investigated and confirmed numerically.

Hence, the deceleration of the speeds in the lower part of the loop seems to be generally observed and seen the discussion about the possible explanations, it does not exclude at all the idea of the observed intensity variations to be falling blobs.

\section{Summary and discussion}

We investigated the EIT shutterless sequence taken on 11 July 2001 in the $304 \AA$ bandpass, and in particular the propagating intensity variations seen along an off-limb half loop. Since there was no evident cause noticable in the direct neighbourhood of the so-called "PD-track", we studied the characteristics of the intensity variations propagating downward in order to interpret them as waves or as a flow. A multi-wavelength analysis of the time frame of the shutterless sequence revealed that the PD loop system was unlikely to be flare-related (although a flare went off south of the analyzed region) and that the bright "blobs" consist of cool plasma since they are only visible in cool temperature bands like $\mathrm{He}$ II and $\mathrm{H} \alpha$.

We analyzed the propagating disturbances in more detail using a method developed by Wiik et al. (1996) in their analysis of intensity variations along off-limb flare loops in $\mathrm{H} \alpha$. Seven "blobs" were identified, propagating down along the loop track with speeds ranging from 30 to $120 \mathrm{~km} \mathrm{~s}^{-1}$, increasing in time. The mean intensity enhancements caused by disturbances range from 9 to $38 \%$ or from 23 to $93 \%$ of the background intensity depending on which background subtraction is carried out.

As a first step in the interpretation of the intensity variations as waves or flows, the parameters found in the analysis are compared with the typical parameters of slow magnetoacoustic waves observed in the solar corona. This comparison led to several anomalies: first, the speed at which the disturbances travel is higher than the local sound speed and secondly, it increases in time while a typical wave propagates with a constant speed. Furthermore, the intensity variations along the PD-track do not show any sign of damping and their amplitudes substantially exceed the intensity enhancements seen in waves. Last but not least, slow waves are only observed propagating upwards while the bright blobs we see all move downwards. Based on the fact that the wave characteristics summed up above are all essential ingredients in the theoretical model developed by Nakariakov et al. (2000) for the observed waves in the atmosphere, we can conclude that the intensity variations along the PD-track are not slow magneto-acoustic waves.

The flow hypothesis, on the other hand, looks more promising. The measured speeds are closely related to the theoretical free-fall speed from a height of $100 \mathrm{Mm}$ and taking into account the uncertainties concerning the projection angle, the curvature of the loop and the exact loop height, this hypothesis looks reasonable. Only in the lower part of the loop, the speeds clearly deviate from the theoretical curves. This effect is observed earlier in almost all speed analyses in flare loops and could be due to plasma deceleration by the loop's helicity, the interaction of the cool downflows with the hotter and denser part of the atmosphere, etc.

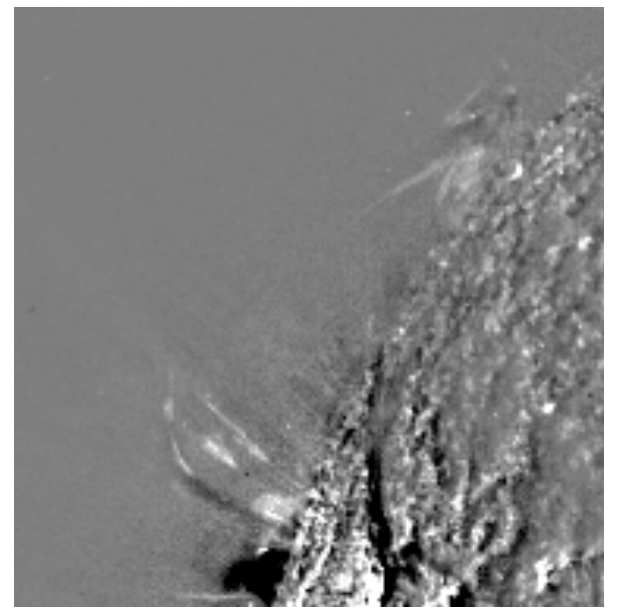

Fig. 12. Part of frame 27 of the EIT-sequence (at 16:32 UT), subtracted by an average image of the sequence to enhance contrast. There is a possible connection between the prominence at the top right of the frame and the PD-track (bottom).

However, despite the similarities with flows, the wave theory cannot be excluded yet. Especially the formation of the blobs is an intriguing problem. It is tempting to think of the quite regularly spaced blobs as a result of some kind of instability or wave. Guided by this idea, Tang et al. (1995) found that bright blobs in flare loops can be created by a sausage instability. This instability is created (only) if the gravitational potential $\Phi$ is large compared to the square of the sound speed (and only for certain wavelengths of the perturbation). However, this theory seems not to hold in our case since the condition $\Phi=G m /(R+z)>v_{\mathrm{s}}^{2}$ is not satisfied for the loop under investigation and still the bright blobs do appear.

Another hypothesis is the idea of kinetic Alfvén waves travelling downward from a reconnection site at the loop top. These trains of kinetic Alfvén waves, propagating with any frequency lower than the ion-cyclotron frequency, might locally enhance the density or temperature and show up as bright features moving down the loop (Voitenko: private communication). The increasing speed could be related to the fact that the phase velocity is proportional to the perpendicular wave number which increases due to phase-mixing. However, one should expect the brightness of the "blobs" to increase closer to the limb and this is not observed. Furthermore, a reconnection event would cause the temperature to rise so that the loop top (and probably also the intensity variations) would be visible in hotter bandpasses which is not the case.

The flow hypothesis raises some questions as well. Where do the blobs come from? They are first visible close to the top of the half loop but how did the extra mass get there? Maybe the loop filled up earlier by the evaporation of chromospheric material following a heating event. But then why is it not observed at higher temperatures? The excess material at the top is most likely cool. Actually, when looking closer to the images in the sequence and the behaviour of the different features seen, it is not impossible that the mass is flowing from the offlimb prominence to the top of the half loop (see Fig. 12). In that case, the PD-track is just one of the channels along which 
the prominence plasma is flowing down (other channels are clearly visible in the sequence movie: some off-limb, north of the PD-track, another channel crosses the solar limb and is visible on disk). We checked $\mathrm{H} \alpha$ data in order to find a cool mass cloud close to the top of the PD-track but preliminary analysis did not show up any connection yet. A thorough investigation of the $\mathrm{H} \alpha$ data is underway and will be presented in the following paper.

Another interesting theory which could explain the origin of the falling mass blobs is the so-called "heating-condensation cycle", first applied by Antiochos et al. (2000) in a prominence configuration and later extended to coronal loops by Müller et al. (2003). It is based on the fact that a magnetic loop which is only heated at the footpoints becomes thermally unstable and rapidly cools down at the apex. Due to the associated pressure drop high up in the loop, mass rises up from the footpoints to the top. The radiative losses increase even further and the collection of mass condensates at the cool loop top and falls down under gravity with increasing speed. When the mass blob encounters the transition region, a rebound shock forms which decelerates the plasma. Finally, the depleted loop reheats and fills up again by chromospheric evaporation and a new cycle starts.

This idea could easily explain the falling bright blobs seen in $304 \AA$. The numerical simulations of Müller et al. (2003) in short, cool loops indeed show plasma blobs falling down in a cyclic pattern, with a clear emission in different transition region lines. The fact that multiple blobs are seen along the PD-track at the same time could be related to the fact that most loops are composed of different (observationally unresolved) threads. The investigation of Müller et al. (2003) will now be extended for loops comparable to the PD-track in the EIT sequence.

Acknowledgements. This work is performed in the framework of the K.U.Leuven project OT/02/57. LvDG acknowledges the Hungarian Government grant OTKA T-038013. For the data, we are grateful to Erwin Verwichte as campaign coordinator of the EIT shutterless sequence of 11 July 2001 and to Vasyl Yurchyshyn for the Big Bear $\mathrm{H} \alpha$ images. Furthermore we thank all the members of the ROB solar group, Yuriy Voitenko and the referee, Ineke De Moortel, for useful discussions and suggestions.

\section{References}

Antiochos, S. K., MacNeice, P. J., \& Spicer, D. S. 2000, ApJ, 536, 494

Aschwanden, M. J. 1987, Sol. Phys., 111, 113

Aschwanden, M. J., Fletcher, L., Schrijver, C. J., \& Alexander, D. 1999, ApJ, 520, 880

Berghmans, D. 1998, Tech. rep., Royal Observatory Belgium, sol.oma.be/High-cadence/data-preprocessing/ index.html

Clette, F. 2000, Tech. rep., Royal Observatory Belgium, sol.oma.be/High-cadence/

De Moortel, I., Hood, A., Ireland, J., \& Walsh, R. 2002a, Sol. Phys., 209, 89

De Moortel, I., Ireland, J., Hood, A., \& Walsh, R. 2002b, A\&A, 387, L13

De Moortel, I., Ireland, J., Walsh, R., \& Hood, A. 2002c, Sol. Phys., 209, 61

Delaboudinière, J.-P., Artzner, G. E., Brunaud, J., et al. 1995, Sol. Phys., 162, 291

Delone, A., Makarova, E., Porfiréva, G., Roschina, E., \& Yukinina, G. 1989, Hvar Observatory Bulletin, 13, 157

Engvold, O., Jensen, E., \& Andersen, B. N. 1979, Sol. Phys., 62, 331

Heinzel, P., Schmieder, B., \& Mein, P. 1992, Sol. Phys., 139, 81

Jensen, E. 1990, in Dynamics of Quiescent Prominences, IAU Colloq., 117,129

Klimchuk, J. A. 2000, Sol. Phys., 193, 53

Loughhead, R. E., \& Bray, R. J. 1984, ApJ, 283, 392

Mein, P., Démoulin, P., Mein, N., et al. 1996, A\&A, 305, 343

Müller, D. A. N., Hansteen, V. H., \& Peter, H. 2003, A\&A, 411, 605

Nakariakov, V. M., Verwichte, E., Berghmans, D., \& Robbrecht, E. 2000, A\&A, 362, 1151

Robbrecht, E., Verwichte, E., Berghmans, D., et al. 2001, A\&A, 370, 591

Roberts, B. R. 2000, Sol. Phys., 193, 139

Tang, Y. H., Fang, C., \& Cui, L. S. 1995, Sol. Phys., 158, 71

Tsiklauri, D., \& Nakariakov, V. M. 2001, A\&A, 379, 1106

Tsubaki, T. 1988, in Solar and Stellar Coronal Structure and Dynamics, 140

Wiik, J. E., Schmieder, B., Heinzel, P., \& Roudier, T. 1996, Sol. Phys., 166,89 